\title{
Electronic structure of InAs/GaSb core-shell nanowires
}

\author{
V. V. Ravi Kishore, ${ }^{*}$ B. Partoens, ${ }^{\dagger}$ and F. M. Peeters ${ }^{\ddagger}$ \\ Department of Physics, University of Antwerp, Groenenborgerlaan 171, 2020 Antwerp, Belgium \\ (Received 29 May 2012; revised manuscript received 19 September 2012; published 23 October 2012)
}

\begin{abstract}
The electronic and optical properties of InAs/GaSb core-shell nanowires are investigated within the effective mass $\mathbf{k} \cdot \mathbf{p}$ approach. These systems have a broken band gap, which results in spatially separated confinement of electrons and holes. We investigated these structures for different sizes of the InAs and GaSb core and shell radius. We found that for certain configurations, the conduction band states penetrate into the valence band states resulting in a negative band gap $\left(E_{g}<0\right)$, which leads to a conduction band ground state that lies below the valence band ground state at the $\Gamma$ point. For certain core-shell wires, only one conduction band state penetrates into the valence band and in this case, a minigap $\Delta$ opens up away from the $\Gamma$ point and as a consequence the electronic properties of the nanowire now depend on both $E_{g}$ and $\Delta$ values.
\end{abstract}

DOI: 10.1103/PhysRevB.86.165439

PACS number(s): 73.21.Hb, 78.20.Bh, 78.67.Lt

\section{INTRODUCTION}

Nanowires with their one-dimensional quantum confinement effect have gathered a lot of attention in the recent past. Growth of single material freestanding nanowires of various materials has been achieved through methods such as molecular beam epitaxy ${ }^{1}$ (MBE) and vapour liquid solid ${ }^{2}$ (VLS) method. Apart from single material nanowires, coreshell nanowires of different combination of materials have also been realized. The latter have resulted in interesting structures, due to their different band alignments. ${ }^{3}$ These structures can be classified into six different types, type I-s, type I-c, type II-s, type II-c, type III-s, and type III-c, ${ }^{3}$ where "c" and "s" represent the structures with electrons confined in core and shell, respectively. These different types have been extensively discussed by Pistol et al. ${ }^{3}$ It was shown that a shell of GaSb around an InAs nanowire core forms a type-III structure, with the $\mathrm{GaSb}$ valence band donating electrons to the conduction band of InAs.

In the present paper, we perform a deeper analysis of such type-III nanowires, where the conduction band minimum of one material is below the valence band maximum of the other and vice versa. Type-III heterostructures have recently gathered a lot of attention due to their enhanced transport properties. They are expected to have large electron densities at high temperatures, ${ }^{4}$ and thus can be used for quantum devices operating at high temperatures. Also heterostructures with antimonide materials have shown interesting electronic properties due to their high mobility. ${ }^{5}$ They can be operated in the $\mathrm{THz}$ regime because of their tunable band structure. ${ }^{6-8}$ Furthermore, they can be used in infrared lasers and detectors. ${ }^{9-11}$ InAs/GaSb superlattices have been studied extensively theoretically ${ }^{12-14}$ as well as experimentally. ${ }^{15-19}$ For example, InAs/GaSb superlattice photodiodes ${ }^{20-23}$ have been reported recently. The electronic ${ }^{24}$ and optical properties ${ }^{25}$ of InAs/GaSb quantum well systems were studied previously, and their behavior in both electric ${ }^{26}$ and magnetic fields ${ }^{27}$ has given important information on spin polarization and the semiconductor-semimetal transition. In InAs/GaSb quantum wells it was found that for certain thicknesses of InAs and GaSb, hybridization ${ }^{13,24,28}$ occurs between electron and hole states, which results in semi-metallic behavior. It was also shown theoretically that a small hybridization gap opens up and the presence of this hybridization gap was later verified experimentally. ${ }^{29,30}$ It is clear that up to now, most attention was given to the electronic properties of $\mathrm{InAs} / \mathrm{GaSb}$ quantum wells and superlattice structures. The above properties of $\mathrm{InAs} / \mathrm{GaSb}$ structures combined with the one dimensional nature of nanowires, can be used in the development of novel electronic devices. Recently, GaSb-InAs(Sb) heterostructure nanowires were studied for tunnel transistor applications. ${ }^{31-33}$ Very recently, also $\mathrm{GaSb} / \mathrm{InAs}(\mathrm{Sb})$ core-shell nanowires were experimentally realized. ${ }^{34}$ It was shown how the carrier concentrations and the transport properties could be tuned from $p$ to $n$ type by changing the shell thickness. Also, ambipolar transport was demonstrated for intermediate shell thickness. To get a better insight into these results, it is essential to understand the electronic and optical properties of these type-III core-shell nanowires, and in the present work, we concentrate on the most promising InAs/GaSb core-shell nanowires.

We use the $\mathbf{k} \cdot \mathbf{p}$ method, which is computationally inexpensive and gives accurate results near the $\Gamma$ point. As the InAs/GaSb core-shell nanowire has a type-III band alignment and as both InAs and GaSb have narrow band gaps, an eightband $\mathbf{k} \cdot \mathbf{p}$ method with Pidgeon and Brown Luttinger-like parameters ${ }^{35}$ is used to calculate the band structure and the optical properties.

In the next section, we discuss the theoretical approach used in this paper. In Sec. III, results of the band structure, the probability density of the valence and the conduction band ground states at $k_{z}=0$, and the absorption spectra for nanowires made of InAs, GaSb, and their core-shell combinations are given. We introduce the hybrid states in these nanowires and study their properties.

\section{THEORY}

In this paper, we consider cylindrical nanowires of InAs, $\mathrm{GaSb}$, and their core-shell nanowires grown in the [001] crystallographic direction. As both InAs and $\mathrm{GaSb}$ are narrow band gap materials where $E_{G} \approx \Delta_{\mathrm{SO}},{ }^{36}$ we use an eightband $\mathbf{k} \cdot \mathbf{p}$ model, which simultaneously describes both the conduction and the valence bands. Both InAs and GaSb have a lattice constant of $6.1 \AA^{16,19}$ and can thus be grown epitaxially, which is the reason why it is a reasonable approximation to 
TABLE I. Band, Luttinger, and Luttinger-like parameters for InAs and $\mathrm{GaSb}$.

\begin{tabular}{lcc}
\hline \hline & InAs & GaSb \\
\hline$E_{G}(\mathrm{eV})$ & 0.37 & 0.81 \\
$E_{G C}(\mathrm{eV})$ & 2.98 & 2.54 \\
$E_{P}(\mathrm{eV})$ & 19.33 & 20 \\
$\Delta(\mathrm{eV})$ & 0.43 & 0.75 \\
$\gamma_{1}$ & 19.81 & 11.8 \\
$\gamma_{2}$ & 8.46 & 4.03 \\
$\gamma_{3}$ & 9.34 & 5.26 \\
$\tilde{\gamma}_{1}$ & 2.3956 & 3.5695 \\
$\tilde{\gamma}_{2}$ & -0.2472 & -0.0852 \\
$\tilde{\gamma}_{3}$ & 0.6328 & 1.1448 \\
\hline \hline
\end{tabular}

neglect strain effects. The band parameters used in this paper are given in Table I. The valence band offset of the InAs/GaSb heterostructure is taken as $\mathrm{VBO}=0.56 \mathrm{eV} .{ }^{37-39}$ Together with the band gap values of Table I, this leads to the band alignment of InAs/GaSb core-shell nanowires as shown in Fig. 1. In this diagram as well as in our calculations, the zero of energy is taken at the conduction band minimum of InAs. The InAs conduction band minimum appears below the valence band maximum of GaSb. This implies that electrons will move from $\mathrm{GaSb}$ to InAs, while free holes will appear in GaSb. The effect of confinement on this charge transfer and thus the band alignment is the topic of this paper. Due to this broken band gap, which results in electron and hole confinement in different regions of the heterostructure, it becomes essential to use at least an eight-band model that includes the effect of both the conduction and the valence band states.

The Luttinger-Kohn (LK) Hamiltonian ${ }^{36}\left(\mathbb{H}_{8}\right)$ is constructed to include the conduction band, the valence band and the band offset (VBO and $\mathrm{CBO}$ ):

$$
\mathbb{H}_{8}=H_{k \cdot p}^{8}+\mathrm{VBO}+\mathrm{CBO} .
$$

The effective electron mass $\left(m^{*}\right)$ and Luttinger-like $\left(\gamma^{L}\right.$ 's) parameters in the core-shell heterostructure can be written as $m^{*}=m_{s}^{*}+\left(m_{c}^{*}-m_{s}^{*}\right)\left[1-\Theta\left(\rho-R_{c}\right)\right]$ and $\gamma^{L}=\gamma_{s}^{L}+$

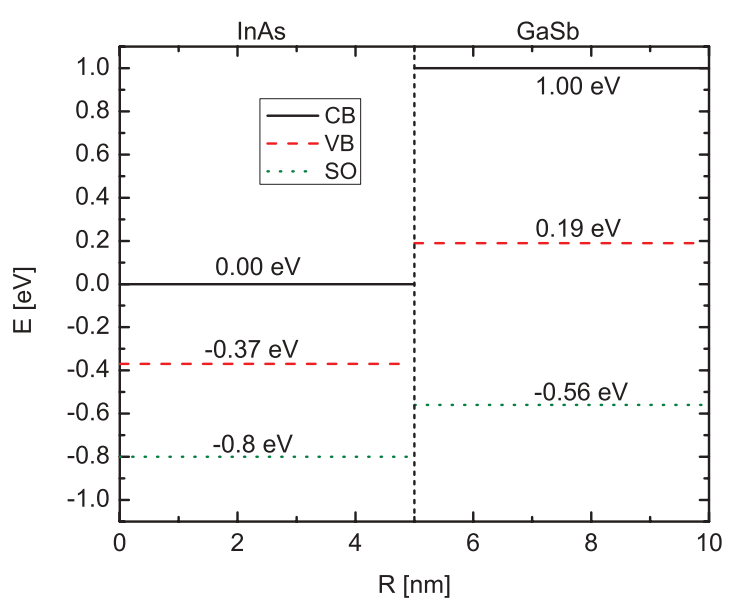

FIG. 1. (Color online) Band alignment of InAs/GaSb core-shell nanowires with core radius $R_{c}=5 \mathrm{~nm}$ and total wire radius $R=10 \mathrm{~nm}$.
$\left(\gamma_{c}^{L}-\gamma_{s}^{L}\right)\left[1-\Theta\left(\rho-R_{c}\right)\right]$, where $m_{s}^{*}$ and $\gamma_{s}$ are the values in the shell and $m_{c}^{*}$ and $\gamma_{c}$ the values in the core, $\Theta$ is the heavy side step function, $\rho$ is the radial coordinate, $R$ is the radius of the total wire, and $R_{c}$ is the radius of the core of the core-shell quantum wire.

The envelope wave function for both conduction and valence bands is expanded in a basis of eigenfunctions of a cylinder with radius $R$ :

$$
\begin{aligned}
& \Psi_{\zeta}^{\sigma}(\rho, \phi, z, \sigma) \\
& =e^{i k_{z}} \sum_{m=-M}^{M} \sum_{l=1}^{L} C_{m l}^{\zeta} \frac{e^{i m \phi}}{\sqrt{2 \pi}} \frac{\sqrt{2}}{R J_{m+1}\left(\alpha_{m l}\right)} J_{m}\left(\alpha_{m l} \frac{\rho}{R}\right) \eta_{\sigma},
\end{aligned}
$$

where $J_{m}$ is the Bessel function of the first kind and $\alpha_{m l}$ is the $l$ th zero of the corresponding Bessel function $J_{m}$ $(m=0, \pm 1, \pm 2, \pm 3, \ldots, \pm M, l=1,2,3, \ldots, L), \zeta$ represents different bands in the eight-band model, i.e., $\zeta \in\{$ el $\uparrow$, el $\downarrow$, hh $\uparrow, \mathrm{lh} \uparrow, \mathrm{lh} \downarrow$, hh $\downarrow$, so $\uparrow$, so $\downarrow\}$ and $R$ is the radius of the nanowire. Here, we have used cylindrical coordinates $(\rho, \phi, z)$. The coefficients $C_{m l}$ are determined by diagonalizing the $\mathbb{H}_{8}$ Hamiltonian. The eigenenergies form a set of subbands numbered by pairs $(m, l)$ and are fourfold degenerate with respect to the spin degree of freedom $(\sigma= \pm)$ and the angular momentum $( \pm m)$. In Eq. (1), the symbol $\eta_{\sigma}$ is the spin part of the wave function. We limited the infinite sum to typically $M=4$ and $L=10$, and we checked that this was sufficient for convergence.

Apart from the band structure obtained by diagonalizing the $\mathbb{H}_{8}$ Hamiltonian, we also calculated the absorption intensity of circular $\left(I_{\sigma}\right)$ and linear $\left(I_{\pi}\right)$ polarized light using the Fermi golden rule. ${ }^{40,41}$ For three different polarizations of light, the integral $I=|\langle f|\vec{p} \cdot \vec{A}| i\rangle|^{2}$ was calculated as follows:

$$
\begin{aligned}
I_{\sigma^{+}} \simeq \mid & \frac{2}{\sqrt{6}}\left\langle\Psi_{\mathrm{el}, \uparrow} \mid \Psi_{h, \frac{3}{2},-\frac{1}{2}}\right\rangle+\frac{2}{\sqrt{2}}\left\langle\Psi_{\mathrm{el}, \downarrow} \mid \Psi_{h, \frac{3}{2},-\frac{3}{2}}\right\rangle \\
& -\left.\frac{2}{\sqrt{3}}\left\langle\Psi_{\mathrm{el}, \uparrow} \mid \Psi_{h, \frac{1}{2},-\frac{1}{2}}\right\rangle\right|^{2}, \\
I_{\sigma^{-}} \simeq & \mid \frac{2}{\sqrt{2}}\left\langle\Psi_{\mathrm{el}, \uparrow} \mid \Psi_{h, \frac{3}{2}, \frac{3}{2}}\right\rangle+\frac{2}{\sqrt{6}}\left\langle\Psi_{\mathrm{el}, \downarrow} \mid \Psi_{h, \frac{3}{2}, \frac{1}{2}}\right\rangle \\
& +\left.\frac{2}{\sqrt{3}}\left\langle\Psi_{\mathrm{el}, \downarrow} \mid \Psi_{h, \frac{1}{2}, \frac{1}{2}}\right\rangle\right|^{2},
\end{aligned}
$$

for circular polarized light and

$$
\begin{aligned}
I_{\pi} \simeq & \mid \sqrt{\frac{2}{3}}\left\langle\Psi_{\mathrm{el}, \downarrow} \mid \Psi_{h, \frac{3}{2},-\frac{1}{2}}\right\rangle+\sqrt{\frac{2}{3}}\left\langle\Psi_{\mathrm{el}, \uparrow} \mid \Psi_{h, \frac{3}{2}, \frac{1}{2}}\right\rangle \\
& +\sqrt{\frac{1}{3}}\left\langle\Psi_{\mathrm{el}, \uparrow} \mid \Psi_{h, \frac{1}{2}, \frac{-1}{2}}\right\rangle+\left.\sqrt{\frac{1}{3}}\left\langle\Psi_{\mathrm{el}, \downarrow} \mid \Psi_{h, \frac{1}{2}, \frac{1}{2}}\right\rangle\right|^{2}
\end{aligned}
$$

for linearly polarized light.

We also study the probability density of conduction and valence band ground states $|\Psi(\rho)|^{2}$, which is given by

$$
\begin{aligned}
|\Psi(\rho)|^{2}= & \left|\Psi_{\mathrm{El}}^{\uparrow}(\rho)\right|^{2}+\left|\Psi_{\mathrm{El}}^{\downarrow}(\rho)\right|^{2}+\left|\Psi_{\mathrm{HH}}^{\uparrow}(\rho)\right|^{2}+\left|\Psi_{\mathrm{LH}}^{\uparrow}(\rho)\right|^{2} \\
& +\left|\Psi_{\mathrm{LH}}^{\downarrow}(\rho)\right|^{2}+\left|\Psi_{\mathrm{HH}}^{\downarrow}(\rho)\right|^{2}+\left|\Psi_{\mathrm{SO}}^{\uparrow}(\rho)\right|^{2}+\left|\Psi_{\mathrm{SO}}^{\downarrow}(\rho)\right|^{2} .
\end{aligned}
$$

From the above relations, it is clear that the density of a particular state in the eight-band model will have contributions 
from both electrons and holes, and depending on the band structure, these contributions can be small or large. For example, in the case of large band gap materials like GaAs, the conduction band ground state will mainly consist of the contribution from the electron states and, similarly, the valence band ground state will mainly have hole contributions. This will no longer be true for small band gap materials like InAs. We discuss in the next section, the above mentioned properties for type-III core-shell nanowires.

\section{RESULTS}

In this section, we present our results for $\mathrm{InAs} / \mathrm{GaSb}$ nanowires, which have a type-III band alignment. The electronic band structure, the density of conduction and valence band ground states at the $\Gamma$ point and the absorption spectra of the electrons and the holes are presented for each case. We first discuss our results for nanowires consisting of a single material, which will be followed by our results for the core-shell nanowires.

\section{A. Single material wires}

Figure 2 gives the band structure, the spinor distribution of both the conduction and the valence band ground states and the absorption spectra for InAs and GaSb nanowires, respectively. Here, the zero of energy is taken at the conduction band minimum of InAs. The results for the valence band structure are shown in Figs. 2(a) and 2(e). Notice that the ground state has a camel back structure for both InAs and $\mathrm{GaSb}$, which results in a peak in the onset of the absorption spectrum. The discontinuities in the spinor distribution of the valence band ground state in Figs. 2(c) and 2(g) are due to the crossings in the corresponding valence band ground state [see Figs. 2(a) and 2(e)]. Notice that linear polarized light has always a lower intensity as compared to circular polarized light. This could be understood from the spinor distribution of the valence band ground state and the relations for calculating the absorption intensity [see Eqs. (2) and (3)]. The spinor distribution of the valence band ground state shows that the light hole $(\mathrm{LH})$ has the maximum contribution close to the zone center, and decreases as we move away from it, whereas the heavy hole $(\mathrm{HH})$ contribution slowly increases as we move away from the zone center. A look at the absorption intensity relations [see Eqs. (2) and (3)] show that the intensity due to circular polarized light $\left(I_{\sigma}=I_{\sigma+}+I_{\sigma-}\right)$, results from the transitions $\mathrm{HH} \rightarrow \mathrm{El}, \mathrm{LH} \rightarrow \mathrm{El}$, and $\mathrm{SO} \rightarrow \mathrm{El}$, while, for linear polarized light $\left(I_{\pi}\right)$, only the transitions $\mathrm{LH} \rightarrow \mathrm{El}$ and $\mathrm{SO} \rightarrow \mathrm{El}$ contribute. Thus $I_{\sigma}$ includes the extra transition $\mathrm{HH} \rightarrow \mathrm{El}$. Furthermore, the lower energy transitions are dominated by $\mathrm{LH} \rightarrow \mathrm{El}$, while the higher energy transitions, which are away from the zone center, are dominated by $\mathrm{HH} \rightarrow$ El. Hence for higher energies there appear many more peaks in $I_{\sigma}$, that are absent in $I_{\pi}$.

The spinor distribution of the valence band ground state [see Figs. 2(c) and 2(g)] shows that at $k_{z}=0$, the light hole gives the largest contribution for both InAs and GaSb. But the spinor distribution of the conduction band ground state [see Figs. 2(b) and 2(f)] shows that at $k_{z}=0$, the heavy hole contributes significantly in the case of InAs which is absent for the larger band gap material GaSb. This can also be seen in the density of the conduction and the valence band ground states in Fig. 3, where the density of the conduction band ground state of InAs [see Fig. 3(a)] shows a small dip near $\rho=0$. A look at the individual components of this density [see Fig. 3(c)] shows that this is due to the contribution of the heavy holes.
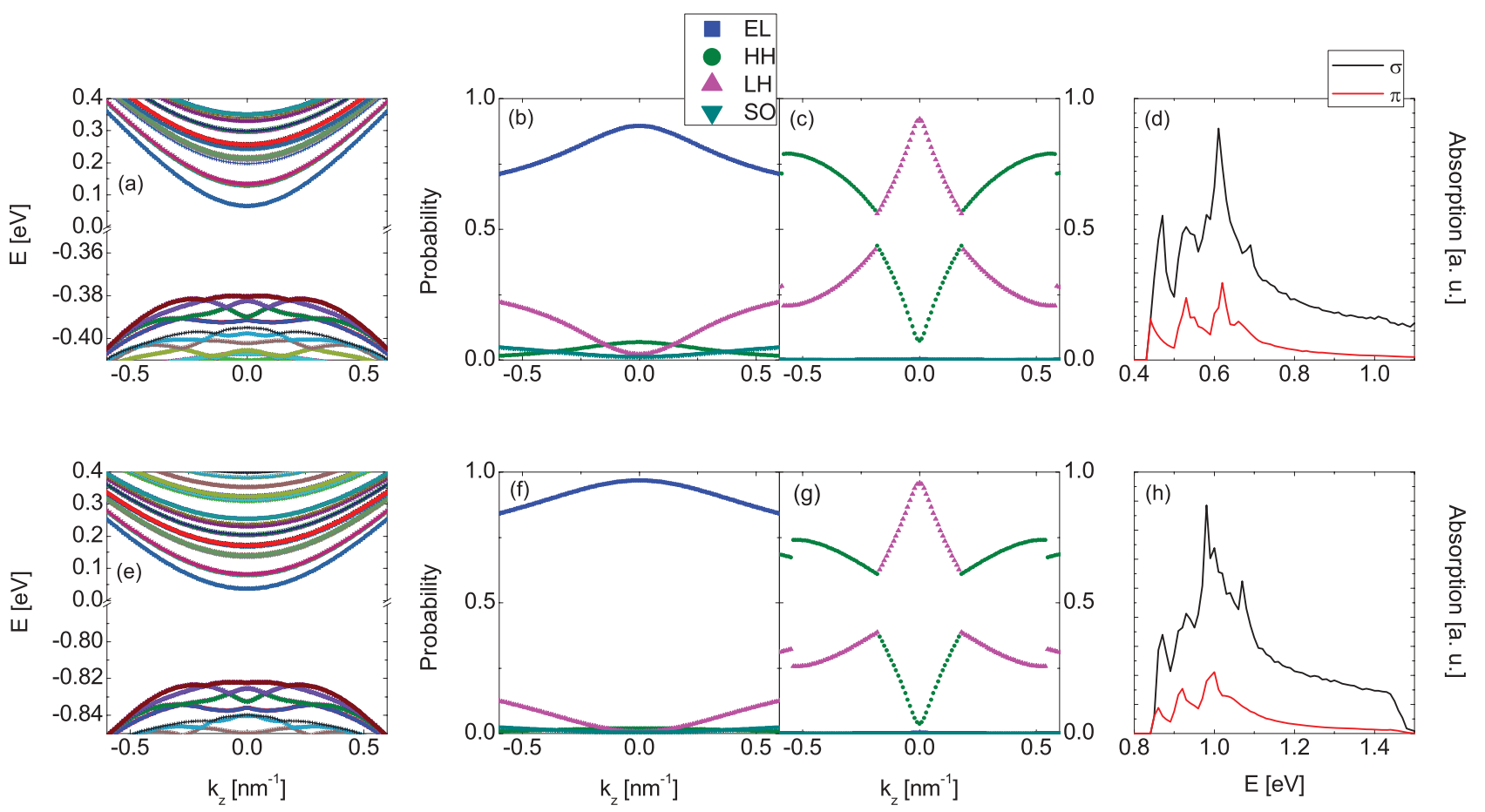

FIG. 2. (Color online) Band structure [(a) and (e)], the spinor distribution of conduction [(b) and (f)], valence band ground states [(c) and (g)], and the optical absorption spectra [(d) and (h)] for InAs and GaSb single material nanowires, respectively, with radius $R=10 \mathrm{~nm}$. 

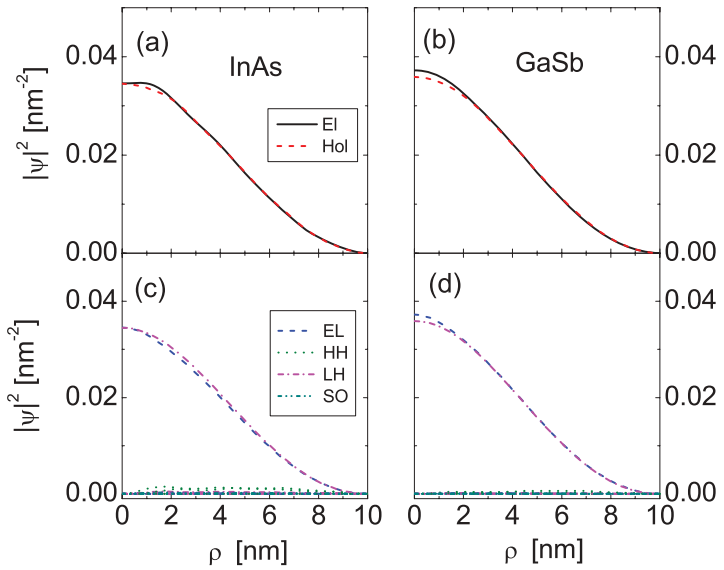

FIG. 3. (Color online) Density of conduction band ground state (black solid lines) and valence band ground state (red solid lines) at $k_{z}=0$ along with the contribution from various states, electrons (dashed), heavy holes (dotted), light holes (dash dot), and split-off (dash dot dot) for the ground state of InAs [(a) and (b)] and GaSb [(c) and (d)] nanowires with radius $R=10 \mathrm{~nm}$.

This increased influence of holes in the conduction band is attributed to the small band gap of InAs, which is $370 \mathrm{meV}$.

\section{B. Core-shell nanowires}

We now present our results for the InAs/GaSb core-shell nanowires. As a first example, we consider a nanowire with core radius $R_{c}=5 \mathrm{~nm}$ and total radius $R=10 \mathrm{~nm}$. Here, the zero of energy is taken at the conduction band minimum of InAs. The band structure of these core-shell nanowires with
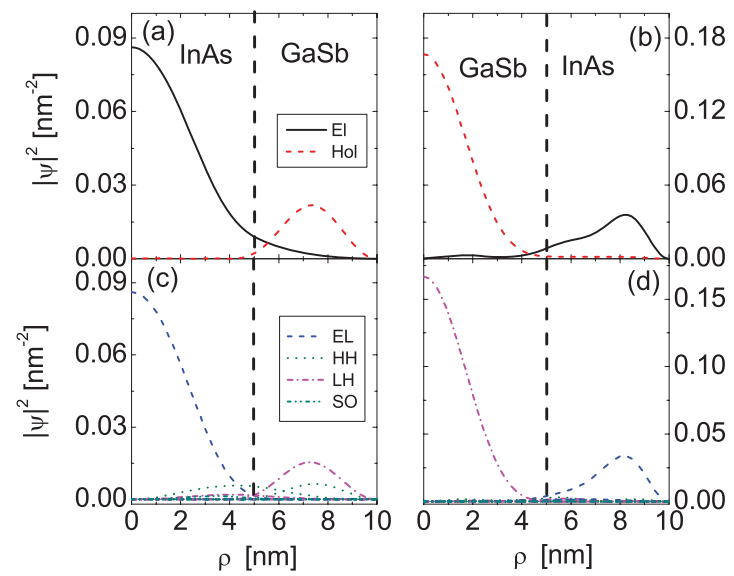

FIG. 5. (Color online) Density of conduction band (black solid lines) and valence band (red solid lines) ground states at $k_{z}=0$ along with the contribution from various states [electron (dashed), heavy holes (dotted), light holes (dash dot), and split-off (dash dot dot)] for the ground state of InAs/GaSb [(a) and (b)] and GaSb/InAs [(c) and (d)] core-shell nanowires with radius $R_{c}=5 \mathrm{~nm}$ and $R=10 \mathrm{~nm}$.

InAs in the core and GaSb in the core are shown in Figs. 4(a) and 4(e), respectively. Despite the band overlap of the bulk materials, the band structure of both these InAs/GaSb and $\mathrm{GaSb} / \mathrm{InAs}$ core-shell nanowires show an effective band gap of 65 and $78 \mathrm{meV}$, respectively. The spinor distribution of the conduction band ground states in both cases show an increase in the heavy hole contribution at $k_{z}=0$, when compared to the results for the pure wires. Also, the spinor distribution of the valence band shows a small contribution of the electron states for nanowires with GaSb in the core.
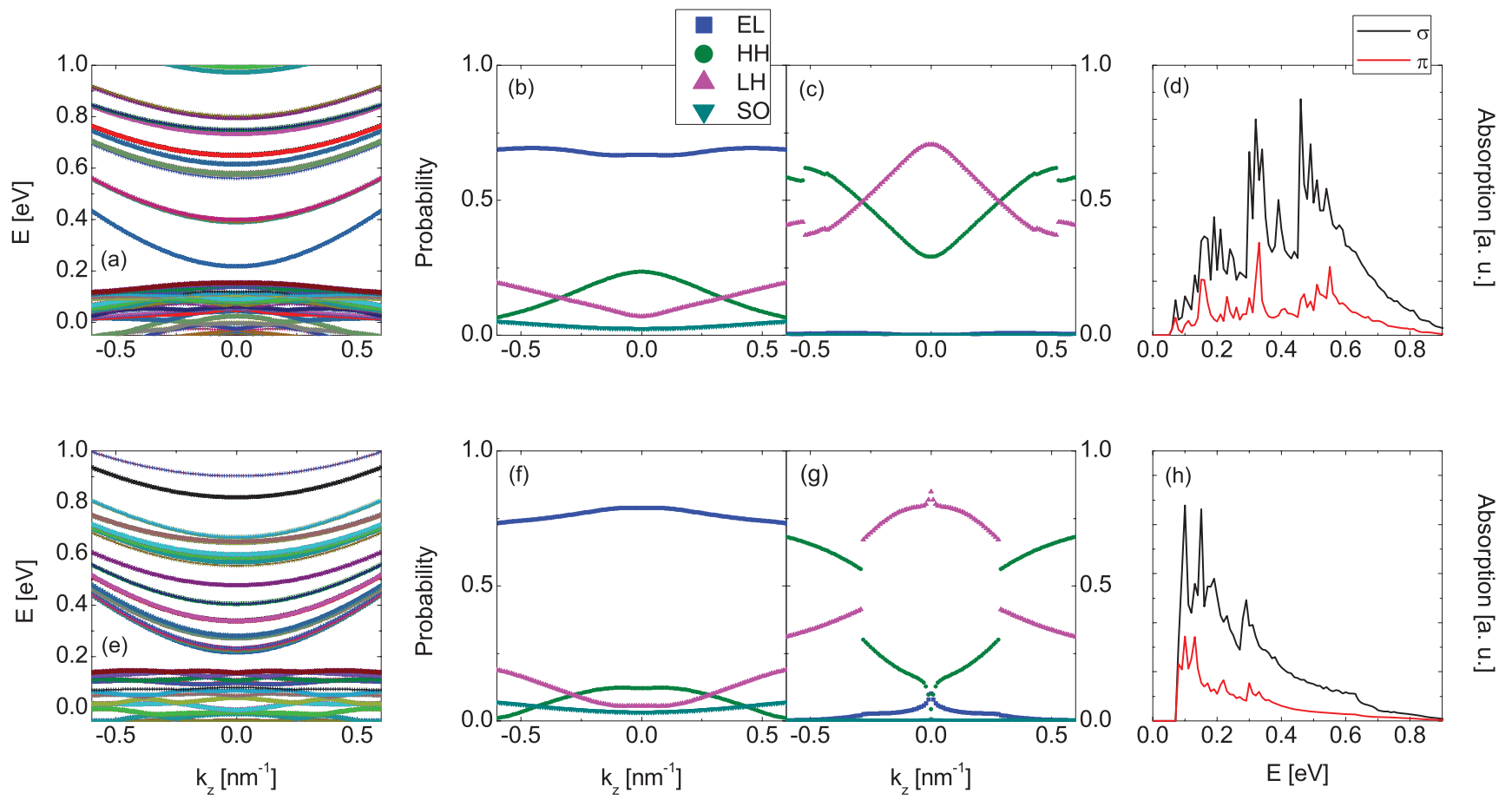

FIG. 4. (Color online) Band structure [(a) and (e)], the spinor distribution of conduction [(b) and (f)] and valence band ground states [(c) and (g)], and the optical absorption spectra [(d) and (h)] for InAs/GaSb and GaSb/InAs core-shell nanowires, respectively, with core radius $R_{c}=5 \mathrm{~nm}$ and total wire radius $R=10 \mathrm{~nm}$. 
For the absorption spectra, the reduced effective band gaps result in absorption at low energies [see Figs. 4(d) and 4(h)]. Absorption peak intensities in the case of nanowires with InAs in the core are reduced because the conduction band state has an increased hole contribution. But at the same time, nanowires with GaSb in the core show an increase in the absorption peak intensity. The reason for this is that the valence band states in this case are spread over large $k_{z}$ values, which results in an increase of equal energy transitions. Finally, we show the probability density of the conduction and the valence band ground states for the core-shell nanowires [see Figs. 5(a) and 5(b)] along with their individual components [see Figs. 5(c) and 5(d)]. The conduction and the valence band ground-state densities are, respectively, confined in the InAs and $\mathrm{GaSb}$ regions, which is due to the particular band alignment (see Fig. 1) in these heterostructures. Although there is an increase in the influence of the hole states in the conduction band density [see Fig. 5(c)], the electron states are still the major contributors and hence we can still label this density as electron density. Similarly, the valence band ground-state density can also be labeled as the hole density.

\section{Electron-hole hybridization}

The effective band gaps can be varied by changing the relative size of the InAs and GaSb regions. Even a negative band gap can be obtained, i.e., when the lowest conduction band state is below the highest valence band state. In this configuration, a hybridization between the conduction band states and the valence band states will occur leading to a semimetallic behavior. ${ }^{42}$ To illustrate this hybridization, we present the band structure of one such hybrid system obtained from an InAs/ $\mathrm{GaSb}$ core-shell nanowire with core radius $R_{c}=6.2$ $\mathrm{nm}$ and total radius $R=18 \mathrm{~nm}$ (see Fig. 6). We have labeled the different states involved in the hybridization from 1 to 4 , where " 1 " is the lowest energy state with a large electron contribution, i.e., the conduction band minimum and "4" is the conduction band state that has a holelike character, i.e., the valence band maximum. Thus we have a negative band gap of $E_{g}=-6.8 \mathrm{meV}$. The negative band gap can result in ambipolar transport as was recently observed in Ref. 34 and in a quantum spin Hall (QSH) insulator phase previously observed in $\mathrm{HgTe} / \mathrm{CdTe}^{43,44}$ and InAs/ $/ \mathrm{GaSb}^{45}$ quantum wells. But Fig. 6 also shows that a new hybridization gap (or minigap) $\Delta=1.3 \mathrm{meV}$ opens up for $k_{z} \neq 0$. Thus we now have a new

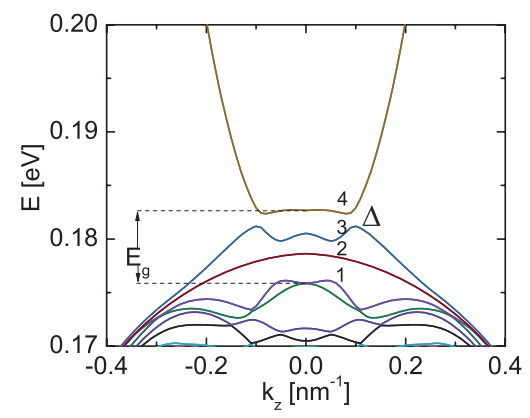

FIG. 6. (Color online) Band structure of InAs/GaSb core-shell nanowire with $R_{c}=6.2 \mathrm{~nm}$ and $R=18 \mathrm{~nm}$.
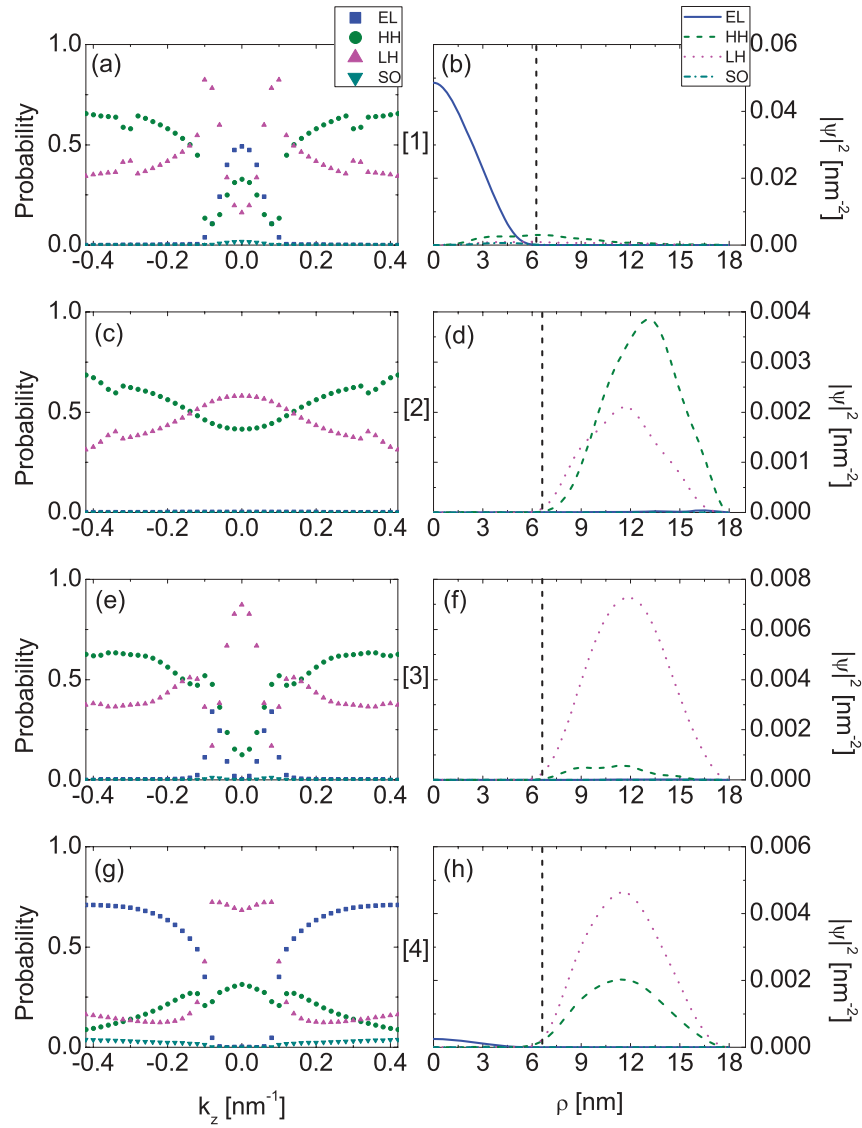

FIG. 7. (Color online) Spinor distribution and particle probability at $k_{z}=0$ of states $1,2,3$, and 4 shown in Fig. 6 for InAs/GaSb core-shell nanowire with $R_{c}=6.2 \mathrm{~nm}$ and $R=18 \mathrm{~nm}$.

gap opening in a semimetallic band dispersion, which results in a bulk insulating behavior for the system.

In Fig. 7, we give the spinor distribution [(a), (c), (e), and $(\mathrm{g})]$ along with the particle probability density at $k_{z}=0$ [(b), (d), (f), and (h)] of the states 1 to 4 . The spinor distribution shows the contribution of the different components at different $k_{z}$ values. It shows that the state " 1 " exhibits a maximum electron contribution, while state " 4 " has a maximum hole

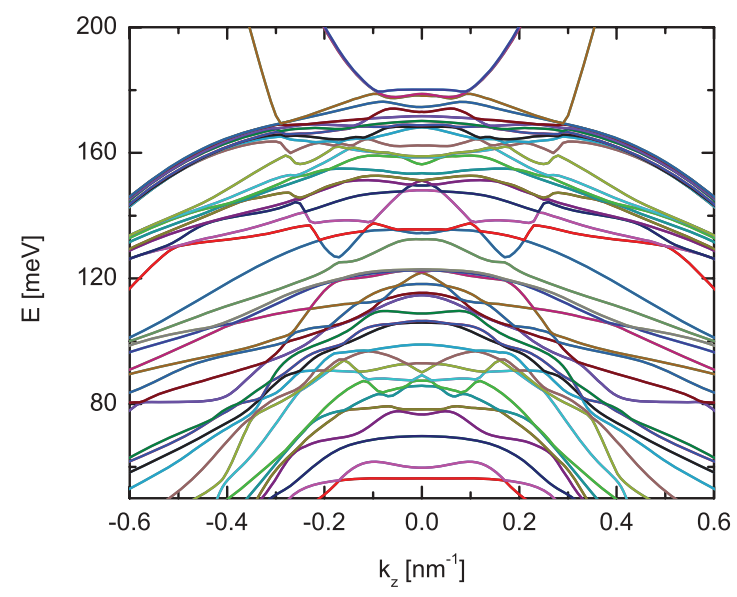

FIG. 8. (Color online) Band structure of an InAs/GaSb core-shell nanowire with $R_{c}=10 \mathrm{~nm}$ and $R=20 \mathrm{~nm}$. 
contribution at $k_{z}=0$. Thus state 4 behaves as a holelike state for $0<k_{z}<0.1 \mathrm{~nm}^{-1}$ and as an electron-like state for $k_{z}>0.1 \mathrm{~nm}^{-1}$. Similarly, state 4 behaves as an electron-like state for $0<k_{z}<0.05 \mathrm{~nm}^{-1}$ and as a holelike state for $k_{z}>0.05 \mathrm{~nm}^{-1}$. This can also be observed from the corresponding probability densities in Figs. 7(b), 7(d), 7(f), and 7(h). The intermediate states 2 and 3 have maximum hole contribution around $k_{z}=0$. Also, it can be seen that the state 4 [see Fig. 7(g)], which is the valence band maximum and state 3 [see Fig. 7(e)] anticross at $k_{z} \simeq 0.1 \mathrm{~nm}^{-1}$. This anticrossing is the origin of the minigap $\Delta$. From the spinor distribution in Figs. 7(e) and 7(g), one can see that the electron and hole contributions are equal at this anticrossing.

A minigap is not always present in case of a band overlap as illustrated in Fig. 8 where we show the band structure of an InAs $/ \mathrm{GaSb}$ core-shell nanowire with $R_{c}=10 \mathrm{~nm}$ and $R=20 \mathrm{~nm}$. Here, we can see that multiple conduction bands penetrate into the valence band states. In this case, there will be multiple hybridization gaps opening at $k_{z} \neq 0$, but not at the same energy values. So, no real minigap $\Delta$ is present in the system. Thus, a real minigap is only present when a single conduction band overlaps with the valence band states at $k_{z}=0$.

In summary for $E_{g}>0$, the InAs/GaSb core-shell nanowire behaves as a semiconductor and for $E_{g}<0$, it behaves as a semimetal. But for $E_{g} \lesssim 0$, the conductivity of the nanowire depends on both the effective bandgap $E_{g}$ and the minigap $\Delta$ values. $^{46}$

Let us now study the ground state of the conduction and the valence bands for InAs/GaSb core-shell nanowires by varying the core radius $R_{c}$ and the total radius $R$. Figure 9 shows the energy of the valence and the conduction band ground states at $k_{z}=0$ for the InAs/GaSb core-shell nanowires as a function of the core radius $R_{c}$, for fixed total radius $R=10$, 20 , and $50 \mathrm{~nm}$. Here, the conduction band (solid black line) and valence band (dashed red line) ground states are shown, for the three different nanowires. In the case of nanowires with $R=10 \mathrm{~nm}$ [see Fig. 9(a)], the effective band gap is always positive and hence there are no hybrid states. This is due to the quantum confinement effect, which increases the effective band gap as the size is reduced (i.e., for small $R)$. Note that there is hybridization for nanowires with radius $R=20 \mathrm{~nm}$ when $6 \mathrm{~nm}<R_{c}<16 \mathrm{~nm}$ and $R=50 \mathrm{~nm}$ when $10 \mathrm{~nm}<R_{c}<45 \mathrm{~nm}$ [see Figs. 9(b) and 9(c)]. In this region of hybridization, the effective band gap is negative. Thus the negative gap $\left(E_{g}\right)$ increases with an increase in $R_{c}$ and reaches a maximum and then decreases, as we increase the size of InAs

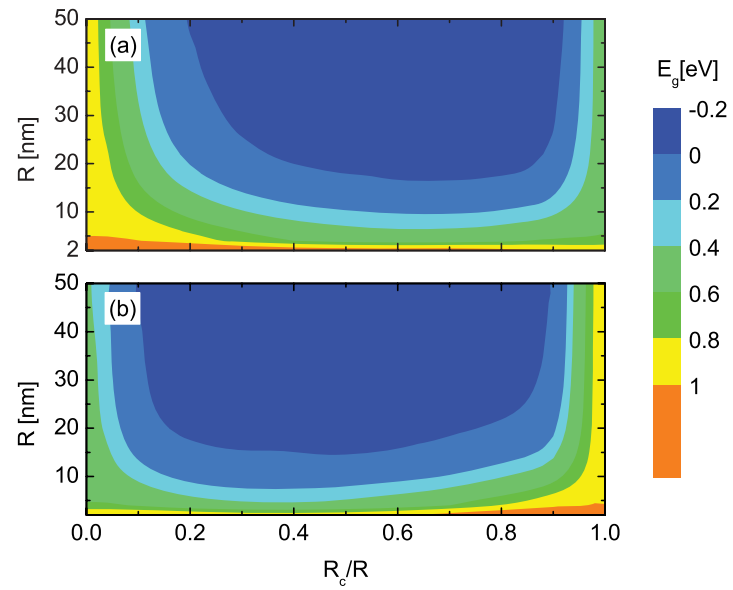

FIG. 10. (Color online) Contour plot of the effective band gap $E_{g}$ at $k_{z}=0$ for (a) InAs/GaSb and (b) GaSb/InAs core-shell wires in the core radius $R_{c} / R$ and total radius $R$ plane.

in these InAs/GaSb core-shell nanowires. Also it is important to highlight that there is a minigap $\Delta$, when $E_{g} \lesssim 0$, where only a single conduction band penetrates into the valence band. This occurs just at the crossing of the conduction and the valence band ground states in Figs. 9(b) and 9(c).

These results can be summarized in a phase diagram in Fig. 10, showing the effective band gap $E_{g}$ as a function of total radius $R$ and $R_{c} / R$ for InAs/GaSb and $\mathrm{GaSb} / \mathrm{InAs}$ coreshell nanowires. We found that a positive band gap is always obtained for $R<14 \mathrm{~nm}$, both for the InAs/GaSb as well as the $\mathrm{GaSb} / \mathrm{InAs}$ core-shell nanowires, irrespective of the core radius $R_{c}$. For $R>14 \mathrm{~nm}$, a minigap and a negative effective band gap can be obtained. Thus by varying the radial size of the InAs/GaSb nanowires we can modify the effective band gap $E_{g}$ and also open a minigap $\Delta$.

As cited in Introduction, in Ref. 34, ambipolar transport was demonstrated in $\mathrm{GaSb} / \mathrm{InAs}(\mathrm{Sb})$ core-shell nanowires, starting from a threshold thickness of about $5 \mathrm{~nm}$ of the $\mathrm{InAs}(\mathrm{Sb})$ shell. We expect the occurrence of ambipolar transport when $E_{g}=0$. For a wire with a GaSb core radius of $R_{c}=25 \mathrm{~nm}$ and InAs shell thickness of $5 \mathrm{~nm}$, we find $E_{g} \simeq-0.05 \mathrm{eV}$. This onset of the closure of the band gap is indeed in good agreement with the experimental observation in Ref. 34.

\section{SUMMARY AND CONCLUSIONS}

In this paper, we have presented different properties of the InAs/GaSb type-III nanowires. Due to the broken band gap
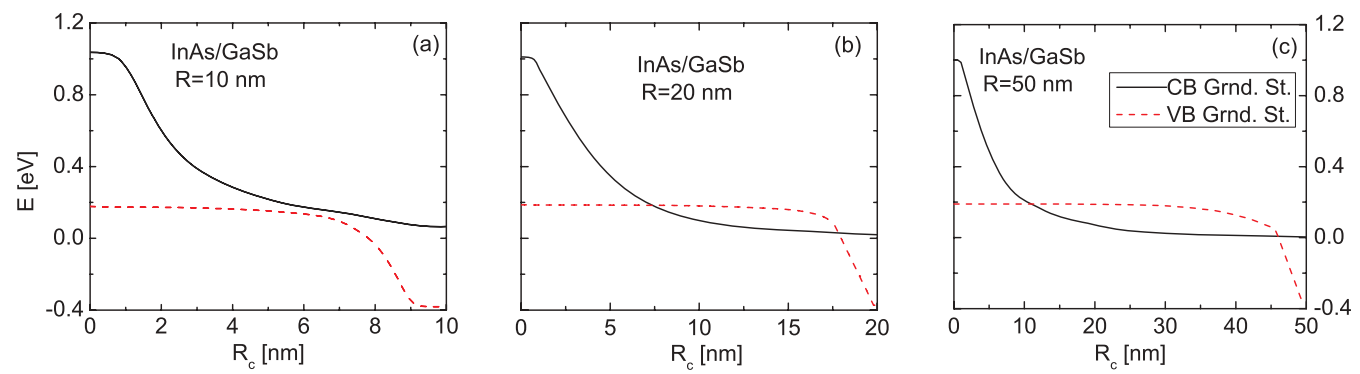

FIG. 9. (Color online) Conduction band minimum and valence band maximum at $k_{z}=0$ for InAs/GaSb core-shell wire as function of the core radius $R_{c}$ with fixed total radius $R=10 \mathrm{~nm}$ (a), $R=20 \mathrm{~nm}$ (b) and $R=50 \mathrm{~nm}$ (c). 
observed in these structures, the electrons and the holes confine in different regions. Apart from this, we have also obtained hybridization of the electron and the hole states, by varying the radius of the total wire $(R)$ and the core region $\left(R_{c}\right)$ of the coreshell nanowires. As the conduction band minimum lies below the valence band maximum, a negative effective band gap $E_{g}<0$ is observed in these systems. Thus these hybridized systems have a semimetallic behavior, which could be used in developing novel electronic devices. We have also observed a small minigap $\Delta$ opening away from the zone center. This minigap can be observed only when a single conduction band overlaps with the valence band states which will influence the conductivity of the system.

\section{ACKNOWLEDGMENT}

This work was supported by the Flemish Science Foundation (FWO-Vl). *ravi.vepakomma@ua.ac.be

†bart.partoens@ua.ac.be

${ }^{\ddagger}$ francois.peeters@ua.ac.be

${ }^{1}$ Z. H. Wu, X. Y. Mei, D. Kim, M. Blumin, and H. E. Ruda, Appl. Phys. Lett. 81, 5177 (2002).

${ }^{2}$ R. S. Wagner and W. C. Ellis, Appl. Phys. Lett. 4, 89 (1964).

${ }^{3}$ M. E. Pistol and C. E. Pryor, Phys. Rev. B 78, 115319 (2008).

${ }^{4}$ M. Inoue, K. Yoh, and A. Nishida, Semicond. Sci. Technol. 9, 966 (1994).

${ }^{5}$ J. B. Boos, B. R. Bennett, N. A. Papanicolaou, M. G. Ancona, J. G. Champlain, R. Bass, and B. V. Shanabrook, Electron. Lett. 43, 834 (2007).

${ }^{6}$ W. Xu, X. F. Wei, and J. Zhang, Appl. Phys. Lett. 92, 162108 (2008). ${ }^{7}$ R. Ascazubi, I. Wilke, K. J. Kim, and P. Dutta, Phys. Rev. B 74, 075323 (2006).

${ }^{8}$ A. J. L. Poulter, M. Lakrimi, R. J. Nicholas, N. J. Mason, and P. J. Walker, Phys. Rev. B 59, 10785 (1999).

${ }^{9}$ H. Ohno, L. Esaki, and E. E. Mendez, Appl. Phys. Lett. 60, 3153 (1992).

${ }^{10}$ H. Mohseni, E. Michel, J. Sandoen, M. Razeghi, W. Mitchel, and G. Brown, Appl. Phys. Lett. 71, 1403 (1997).

${ }^{11}$ G. Liu and S. L. Chuang, Phys. Rev. B 65, 165220 (2002).

${ }^{12}$ G. A. Sai-Halasz, L. Esaki, and W. A. Harrison, Phys. Rev. B 18, 2812 (1978).

${ }^{13}$ M. Altarelli, Phys. Rev. B 28, 842 (1983).

${ }^{14}$ Y. C. Chang and J. N. Schulman, Phys. Rev. B 31, 2069 (1985).

${ }^{15}$ T. Andlauer and P. Vogl, Phys. Rev. B 80, 035304 (2009).

${ }^{16}$ F. Wang, Y. Jia, S. F. Li, and Q. Sun, J. Appl. Phys. 105, 043101 (2009).

${ }^{17}$ S. B. Rejeb, M. Debbichi, M. Sai, A. Gassenq, E. Tournie, and P. Christol, J. Phys. D: Appl. Phys. 43, 325102 (2010).

${ }^{18}$ B. Montanari, M. Peressi, S. Baroni, and E. Molinari, Appl. Phys. Lett. 69, 3218 (1996).

${ }^{19}$ H. Kroemer, Physica E 20, 196 (2004).

${ }^{20}$ H. Mohseni, M. Razeghi, G. J. Brown, and Y. S. Park, Appl. Phys. Lett. 78, 2107 (2001).

${ }^{21}$ A. Gin, Y. Wei, A. Hood, A. Bajowala, V. Yazdanpanah, M. Razeghi, and Tidrow, Appl. Phys. Lett. 84, 2037 (2004).

${ }^{22}$ Y. Wei, A. Hood, A. Bajowala, V. Yazdanpanah, M. Razeghi, Tidrow, and V. Nathan, Appl. Phys. Lett. 86, 091109 (2005).

${ }^{23}$ S. Mallick, K. Banerjee, S. Ghosh, E. Plis, J. B. Rodriguez, S. Krishna, and C. Grein, Appl. Phys. Lett. 91, 241111 (2007).

${ }^{24}$ A. Zakharova, S. T. Yen, and K. A. Chao, Phys. Rev. B 64, 235332 (2001).
${ }^{25}$ E. Halvorsen, Y. Galperin, and K. A. Chao, Phys. Rev. B 61, 16743 (2000).

${ }^{26}$ A. Zakharova, I. Lapushkin, K. Nilsson, S. T. Yen, and K. A. Chao, Phys. Rev. B 73, 125337 (2006).

${ }^{27}$ A. Zakharova, S. T. Yen, and K. A. Chao, Phys. Rev. B 69, 115319 (2004).

${ }^{28}$ K. Nilsson, A. Zakharova, I. Lapushkin, S. T. Yen, and K. A. Chao, Phys. Rev. B 74, 075308 (2006).

${ }^{29}$ M. J. Yang, C. H. Yang, B. R. Bennett, and B. V. Shanabrook, Phys. Rev. Lett. 78, 4613 (1997).

${ }^{30}$ M. Lakrimi, S. Khym, R. J. Nicholas, D. M. Symons, F. M. Peeters, N. J. Mason, and P. J. Walker, Phys. Rev. Lett. 79, 3034 (1997).

${ }^{31}$ B. M. Borg, K. A. Dick, B. Ganjipour, M. E. Pistol, L. E. Wernersson, C. Thelander, and L. Esaki, Nano Lett. 10, 4080 (2010).

${ }^{32}$ M. Ek, B. M. Borg, A. W. Dey, B. Ganjipour, C. Thelander, L. E. Wernersson, and K. A. Dick, Cryst. Growth Des. 11, 4588 (2011).

${ }^{33}$ B. Ganjipour, A. W. Dey, B. M. Borg, M. Ek, M. E. Pistol, K. A. Dick, L. E. Wernersson, and C. Thelander, Nano Lett. 11, 4222 (2011).

${ }^{34}$ B. Ganjipour, M. Ek, B. M. Borg, K. A. Dick, M. E. Pistol, L. E. Wernersson, and C. Thelander, Appl. Phys. Lett. 101, 103501 (2012).

${ }^{35}$ C. R. Pidgeon and R. N. Brown, Phys. Rev. 100, 575 (1966).

${ }^{36}$ V. V. Ravi Kishore, N. Čukarić, B. Partoens, M. Tadić, and F. M. Peeters, J. Phys.: Condens. Matter 24, 135302 (2012).

${ }^{37}$ A. J. Ekpunobi, Mat. Sci. Sem. Proc. 8, 463 (2005).

${ }^{38}$ A. Sai-Halasz, L. L. Chang, J.-M. Welter, C.-A. Chang, and L. Esaki, Solid State Commun. 27, 935 (1978).

${ }^{39}$ L. M. Claessen, J. C. Maan, M. Altarelli, P. Wyder, L. L. Chang, and L. Esaki, Phys. Rev. Lett. 57, 2556 (1986).

${ }^{40}$ P. Y. Yu and M. Cardona, in Fundamentals of Semiconductors, 3rd ed. (Springer, New York, 2003), p. 260.

${ }^{41}$ V. V. Ravi Kishore, B. Partoens, and F. M. Peeters, Phys. Rev. B 82, 235425 (2010)

${ }^{42}$ L. L. Chang and L. Esaki, Surf. Sci. 98, 70 (1980).

${ }^{43}$ B. A. Bernevig, T. L. Hughes, and S. C. Zhang, Science 314, 1757 (2006).

${ }^{44}$ M. Konig, S. Wiedmann, C. Brune, A. Roth, H. Buhmann, L. W. Molenkamp, X. L. Qi, and S. C. Zhang, Science 318, 766 (2007).

${ }^{45}$ C. Liu, T. L. Hughes, X. L. Qi, K. Wang, and S. C. Zhang, Phys. Rev. Lett. 100, 236601 (2008).

${ }^{46}$ I. Knez, R. R. Du, and G. Sullivan, Phys. Rev. B 81, 201301 (2010). 